Carnets de géographes

GÉOGRAPHES.

\title{
Conditions de vie et projets migratoires des réfugiés libériens à Conakry (Guinée) et Accra (Ghana)
}

\section{Hélène Simon-Lorière}

\section{CpenEdition}

Journals

Édition électronique

URL : http://journals.openedition.org/cdg/490

DOI : $10.4000 / \mathrm{cdg} .490$

ISSN : 2107-7266

Éditeur

UMR 245 - CESSMA

\section{Référence électronique}

Hélène Simon-Lorière, "Conditions de vie et projets migratoires des réfugiés libériens à Conakry (Guinée) et Accra (Ghana) », Carnets de géographes [En ligne], 7 | 2014, mis en ligne le 01 décembre 2014, consulté le 23 septembre 2020. URL : http://journals.openedition.org/cdg/490 ; DOI : https:// doi.org/10.4000/cdg.490

\section{cc)}

La revue Carnets de géographes est mise à disposition selon les termes de la Licence Creative Commons Attribution - Pas d'Utilisation Commerciale - Pas de Modification 4.0 International. 


\title{
CONDITIONS DE VIE ET PROJETS MIGRATOIRES DES RÉFUGIÉS LIBÉRIENS
}

\author{
À CONAKRY (GUINÉE) ET ACCRA (GHANA)
}

\section{HÉLÈNE SIMON-LORIÈRE}

Cette thèse de géographie s'est intéressée à la situation en 2008-2009 des réfugiés libériens dans deux capitales d'Afrique de l'Ouest, Conakry en Guinée et Accra au Ghana. À l'issue du conflit au Libéria, elle pose la question de l'avenir de ces réfugiés urbains dans ces deux pays proches du Libéria où ils avaient trouvé refuge entre 1990 et 2003. Dans une perspective longitudinale, elle suit la problématique suivante : les réfugiés rencontrés ont-ils transformé leur expérience de migration forcée en une migration ou une sédentarité assumée ?

\section{Une vision longitudinale des expériences d'exil des Libériens}

Le plan de la thèse adopte une vision rétrospective et prospective. La première partie est centrée sur le contexte de l'asile libérien : elle apporte des éléments de compréhension du conflit civil dans ce pays puis traite de la gestion des réfugiés par les « autorités de l'asile ». La deuxième partie éclaire le parcours migratoire et le quotidien des réfugiés en exil. Ce dernier est analysé au prisme de la notion de reterritorialisation en restituant l'organisation spatiale des réfugiés dans chaque agglomération et leurs modes de subsistance. Enfin, la dernière partie explore les perspectives migratoires des Libériens, mettant en avant le décalage dans le temps entre les «solutions durables» du HCR (rapatriement volontaire, intégration locale, réinstallation) et les «tactiques migratoires post-conflit » individuelles et collectives.

\section{Au carrefour des géographies ubaine, sociale, politique et des migrations}

Inscrite dans le champ des Forced Migration Studies, cette thèse aborde les expériences des réfugiés dans un continuum analytique qui déconstruit la dichotomie classique entre les catégories de réfugié et migrant. Elle propose une radioscopie d'un conflit africain peu connu du monde francophone, celui du Libéria, replacé dans la dynamique sous régionale des conflits de la Mano River. Enfin, elle met l'accent sur «l'agencéité » des réfugiés, leurs marges de manœuvre et leurs choix possibles, si ténus soient-ils. Si les contextes d'asile locaux, notamment urbains, et internationaux, liés aux politiques migratoires mises en œuvre pour les Libériens par la communauté internationale, sont pris en considération, l'approche privilégie le point de vue des réfugiés, leur appréhension des règles du jeu migratoire et leurs façons de «faire avec ». Sont ainsi articulées géographie des migrations, géographie politique et géographie sociale. La dimension urbaine de la thèse réside dans la comparaison entre un camp de réfugiés en marge de l'agglomération capitale au Ghana, celui de Buduburam près d'Accra, et une situation de dispersion dans la capitale guinéenne, en l'absence de camp à 
Conakry. La question classique de l'insertion urbaine est ainsi travaillée à partir de ces deux configurations spatiales.

\section{Une méthodologie qualitative}

Pour pouvoir produire un discours analytique sur la situation des réfugiés libériens, des entretiens à géométrie variable ont été menés auprès de 250 personnes, réfugiées statutaires ( $80 \%$ ) ou non, pour les $4 / 5^{\mathrm{e}}$ dans les différentes communes de l'agglomération de Conakry, pour $1 / 5^{\mathrm{e}}$ dans le camp de Buduburam et dans des districts d'Accra et de la périphérie de la capitale ghanéenne. L'abondante littérature sur ce camp de réfugiés a permis de compléter ce deuxième terrain et de compenser cette dissymétrie. Cette dernière est par ailleurs assumée, en lien avec la «fabrique » du terrain par laquelle Conakry a été le point d'entrée dans ce sujet de recherche, mais surtout en lien avec la volonté de documenter une situation de refuge hors camp. Les observations des conditions de vie prennent donc une part importante dans la thèse, au côté de la restitution d'extraits d'entretiens en anglais. Ces témoignages de réfugiés ont été complétés par la collecte d'informations sur le contexte de l'asile en Guinée et au Ghana : par la mobilisation d'archives de presse et par des entretiens auprès d'acteurs politiques, locaux et nationaux, et humanitaires.

\section{Les réseaux sociaux comme outil d'enquête...}

La présence du camp à Accra a entrainé un accès institutionnalisé aux réfugiés, car les entretiens qui y ont été conduits par l'intermédiaire du comité des réfugiés. À Conakry, en revanche, la dispersion des réfugiés, le manque de légitimité du comité des réfugiés ainsi que les efforts de ne pas apparaitre comme «embarquée» au sein du HCR, ont entrainé la mobilisation des réseaux sociaux pour obtenir des entretiens. Comme le montrent le schéma et la carte ci-dessous, la méthode boule de neige a permis de multiplier les contacts : à partir d'une restauratrice de rue guinéenne, mais surtout de son client habituel libérien, Joseph R, sur le schéma, une cinquantaine de personnes, résidant ou travaillant dans différents secteurs de Conakry ont ainsi été interviewés. Cette méthode a également été employée, à moins grande échelle, pour effectuer des entretiens au Ghana, en dehors du camp de Buduburam.

\section{... et comme outil d'analyse}

Les liens et interactions entre Libériens ont permis d'identifier des enquêtés, mais aussi de saisir des stratégies, individuelles et collectives, pour la survie quotidienne autant pour des perspectives d'avenir. Ils sous-tendent plusieurs analyses dans la thèse : sur l'organisation interne des réfugiés à Conakry et à Accra et leurs façons de «faire groupe », notamment face au HCR ; sur la dispersion des Libériens suite au conflit et au changement de la composition et de la spatialisation de la diaspora libérienne; sur l'émergence ou la résurgence de circulations transnationales entre le Libéria et les anciens pays d'asile puisque les Libériens ont perdu leur statut de réfugié en 2012. 
Figure 1 Les réfugiés rencontrés à partir du premier contact avec Joseph $R$. [CKY $\mathbf{N}^{\circ} 001$ ]

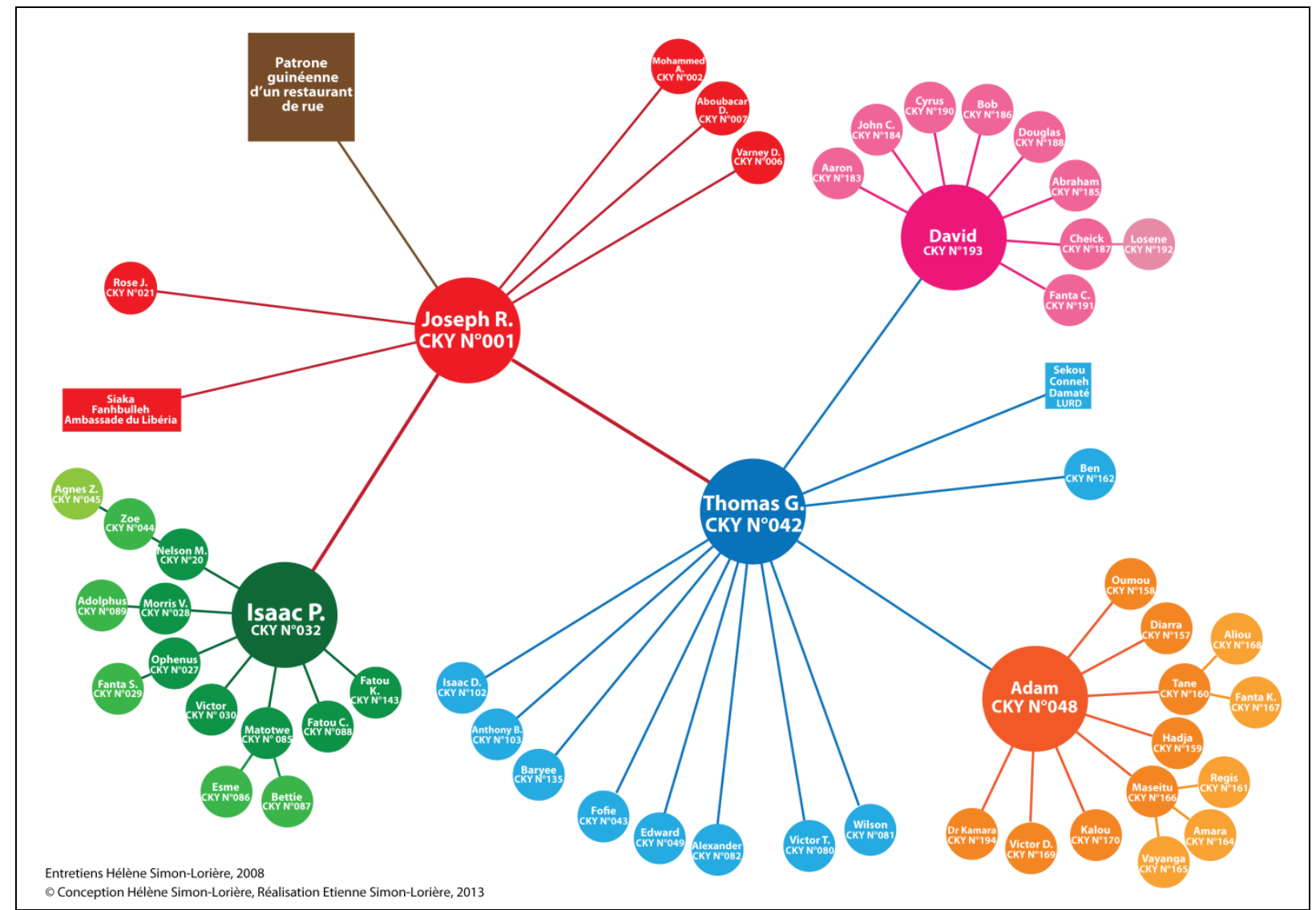

Figure 2 Localisation des entretiens rendus possibles par Joseph R. [CKY $\mathbf{N}^{\circ}$ 001]

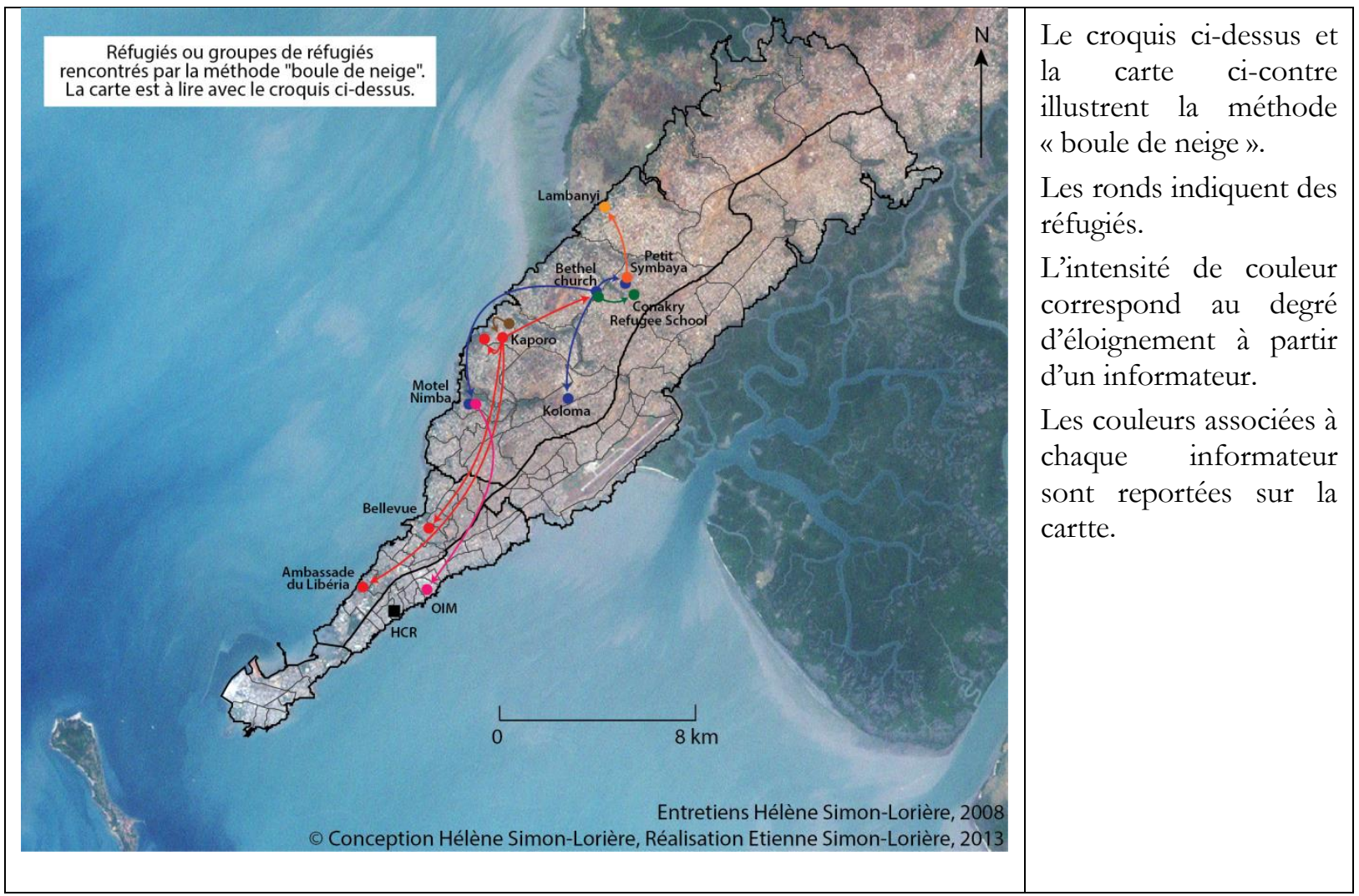


Fiche informative

Lien électronique si la thèse est disponible en ligne

http://tel.archives-ouvertes.fr/tel-00959961

\section{Discipline}

Géographie

\section{Directeur}

Véronique LASSAILLY-JACOB, Monique BERTRAND

\section{Université}

Université de Poitiers

Membres du jury de thèse, soutenue le 13 décembre 2013

Monique BERTRAND, Directrice de recherche IRD (directrice)

Mohamed KAMEL DORAÏ, Chargé de recherche CNRS (examinateur)

Catherine FOURNET-GUERIN, Maître de Conférences HDR, Université de Reims Champagne Ardenne (rapporteur)

France GUERIN-PACE, Directrice de recherche INED (présidente du jury)

Véronique LASSAILLY-JACOB, Professeur émérite, Université de Poitiers (directrice)

Marc-Antoine PEROUSE DE MONTCLOS, Professeur, Université Paris 8 (rapporteur)

\section{Situation professionnelle actuelle}

ATER, Université Paris Sorbonne, Paris IV

Membre du laboratoire Migrinter - UMR 7301 CNRS / Université de Poitiers

\section{Courriel de l'auteur}

hsimonloriere7@yahoo.fr 